\title{
Isolated Internal Auditory Canal Diverticula: A Normal Anatomic Variant Not Associated with Sensorineural Hearing Loss
}

\author{
(DD.C. Mihal, (D)Y. Feng, (D) M.L. Kodet, (DC.M. Lohse, (D) M.L Carlson, and ID.I. Lane
}

\begin{abstract}
BACKGROUND AND PURPOSE: Bony internal auditory canal diverticula are relatively common, occurring in approximately $5 \%$ of temporal bone CTs. Internal auditory canal diverticula have historically been considered incidental; however, a recent publication reported that internal auditory canal diverticula are associated with sensorineural hearing loss. The objective of this study was to further characterize this potential association in a large cohort of patients.
\end{abstract}

MATERIALS AND METHODS: A total of 1759 patients undergoing high-resolution temporal bone CT were collected during a 6-year interval, and audiometric data were obtained from those with internal auditory canal diverticula. To assess any association of isolated internal auditory canal diverticula with sensorineural hearing loss, we excluded from further analysis patients with concomitant otosclerosis and bilateral diverticula and those without audiometric data, leaving 22 index cases. Audiometric data for the ear with a diverticulum was compared with that in the contralateral ear, to serve as an internal control.

RESULTS: Of 1759 patients, $82(4.7 \%)$ had either unilateral $(n=33,40 \%)$ or bilateral $(n=49,60 \%)$ internal auditory canal diverticula. The co-incidence of otosclerosis and internal auditory canal diverticula was $34 \%(n=28)$. There was no correlation between patient age and diverticulum size on either side. Among the index cases with isolated unilateral internal auditory canal diverticula and complete audiometric data, word recognition scores and the prevalence and severity of sensorineural hearing loss were not significantly different comparing the internal auditory canal diverticulum side to its contralateral control.

CONCLUSIONS: This study did not find a statistically significant association between ears with internal auditory canal diverticula and worsening sensorineural hearing loss or word recognition. Internal auditory canal diverticula most likely represent a normal anatomic variant in ears without otosclerosis.

ABBREVIATIONS: $\mathrm{CHL}=$ conductive hearing loss; $\mathrm{HL}=$ hearing loss; $\mathrm{IAC}=$ internal auditory canal; $\mathrm{IQR}=$ interquartile range, $\mathrm{MHL}=$ mixed hearing loss; $\mathrm{SNHL}=$ sensorineural hearing loss

$A^{\prime}$ $\mathrm{n}$ internal auditory canal (IAC) diverticulum (Fig 1) is a focal, well-demarcated lucency in the bone along the anteroinferior margin of the IAC fundus that may occur in cavitary otosclerosis or in isolation. This has also been referred to as "cupping" of the IAC in previous publications (Fig 2). ${ }^{1,2}$ IAC diverticula are relatively common, observed in approximately $5 \%-10 \%$

Received April 20, 2018; accepted after revision September 16.

From the Departments of Radiology (D.C.M., J.I.L.), Otolaryngology-Head and Neck Surgery (Y.F., M.L.K., M.L.C.), and Health Sciences Research (C.M.L.), Mayo Clinic, Rochester, Minnesota. Dr Mihal is currently affiliated with the Cleveland Clinic, Cleveland, Ohio.

Abstract previously presented at: Annual Meeting of the American Society of Neuroradiology and the Foundation of the ASNR Symposium, June 2-7, 2018; Vancouver, British Columbia, Canada.

Please address correspondence to John (Jack) I. Lane, MD, Department of Radiology, Mayo Clinic, 200 First St SW, Rochester, MN 55905; e-mail: Lane.John@mayo.edu

http://dx.doi.org/10.3174/ajnr.A5862 of those undergoing high-resolution temporal bone CT. ${ }^{1,3,4}$ More recently, IAC diverticula have been reported to be associated with sensorineural hearing loss (SNHL) with or without concurrent otosclerosis. ${ }^{3,4}$ The purpose of this study was to elucidate whether isolated IAC diverticula are more commonly encountered as incidental findings as suggested by the pathologic literature or indicative of a pathologic process associated with SNHL.

\section{MATERIALS AND METHODS Imaging Review}

A retrospective review of all temporal bone CTs obtained during a nearly 6-year period (June 9, 2009, to March 8, 2015) in patients with research-use approval was performed by a single radiologist with a Certificate of Added Qualification in neuroradiology and $>20$ years of clinical experience. All patients with a focal lucency in the anteroinferior margin of the IAC fundus (IAC diverticulum) in one or both ears were collected into a database. Each 
temporal bone CT scan in this cohort was obtained on CT scanners from the same manufacturer Siemens Definition and Siemens Flash (Siemens, Erlangen, Germany) with either 64- or 128-detector rows/slices. Measurement of the anteroposterior depth for each IAC diverticulum was recorded in addition to the presence of radiologic evidence of fenestral and retrofenestral otosclerosis. The indications for each study were also recorded, and the medical history was reviewed for the presence of clinical concern for hearing impairment at the time the CT scan was ob- tained. Secondarily, available MR images of the head in the 82 patients with CT findings of IAC diverticula were reviewed to assess for the presence of fluid signal within the diverticula.

\section{Audiometry Review}

All available audiometric data were collected and consisted of air conduction and bone conduction thresholds at set frequencies of 250, 500, 1000, 2000, 3000, and $4000 \mathrm{~Hz}$, calculated 4-frequency pure tone averages $(500,1000,2000,4000)$, air-bone gap, word recognition scores, and a designation of SNHL, conductive hearing loss (CHL), mixed hearing loss (MHL), or normal hearing.

\section{Statistical Analysis}

Continuous features were summarized with means and SDs when approximately normally distributed and with medians and interquartile ranges (IQRs) otherwise; categoric features were summarized with frequency counts and percentages. IAC diverticula sizes were compared between patients with and without otosclerosis using a Wilcoxon rank sum test. Potential associations between patient age and diverticulum size were evaluated using Spearman rank correlation coefficients. Comparisons of hearing classification and audiometric data between ears with and without IAC diverticula in the same patient were diverticula (black and white arrows) in the same patient with unilateral hearing loss.

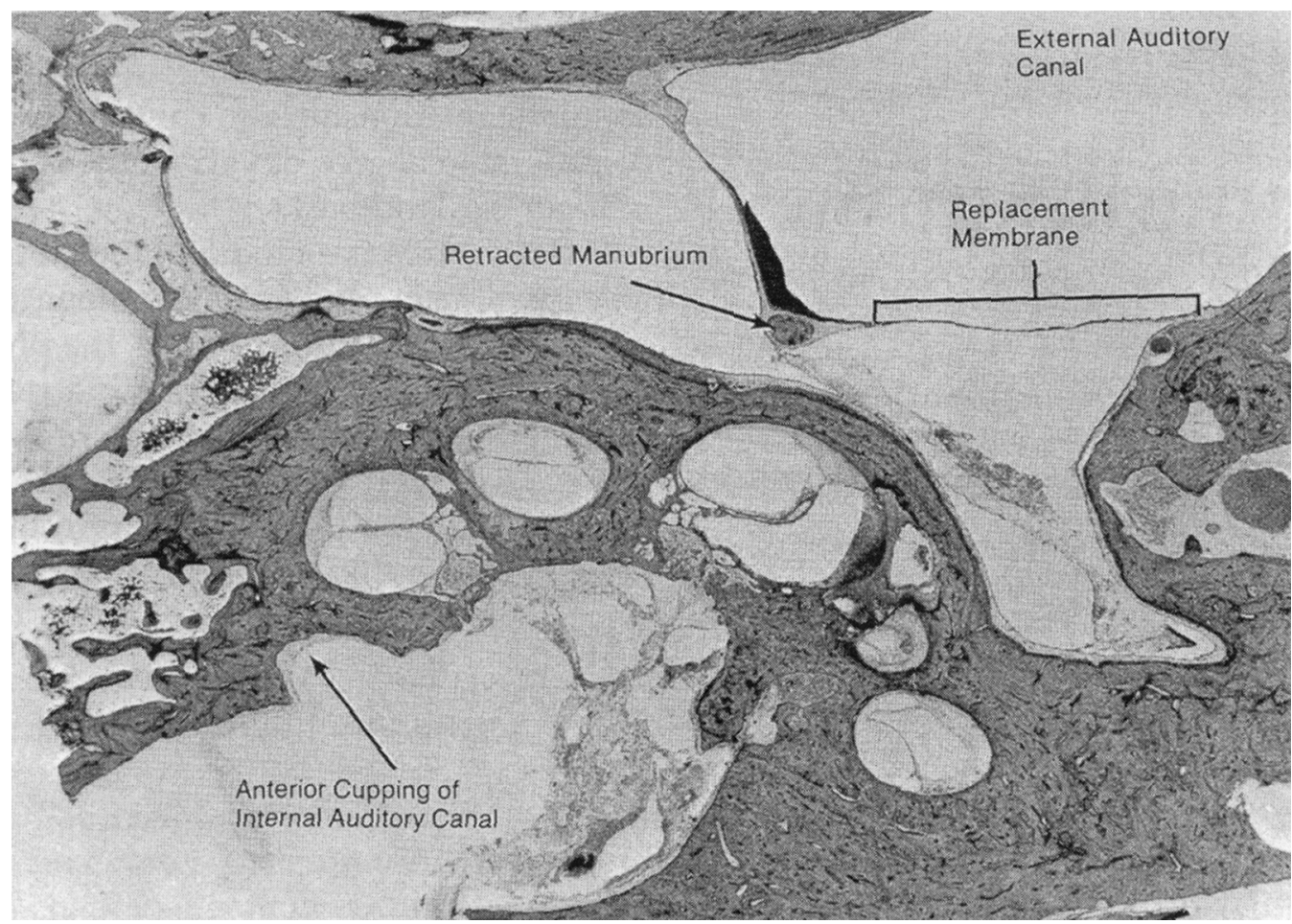

FIG 2. Histologic micrograph demonstrating the typical location of IAC diverticulum (ie, IAC cupping). Reproduced with permission from the third edition of Guyla and Schucknecht's Anatomy of the Temporal Bone with Surgical Implications. ${ }^{1}$ 
evaluated using the McNemar and Wilcoxon signed rank tests to account for the paired nature of the data. Statistical analyses were performed using Version 9.4 of the SAS software package (SAS Institute; Cary, North Carolina). All tests were 2-sided, and $P$ values $<.05$ were considered statistically significant.

\section{RESULTS}

A total of 1759 patients were scanned during the nearly 6-year study interval. Eighty-two of these patients (4.7\%) had a focal lucency along the anteroinferior margin of the IAC fundus unilaterally $(n=33,40 \%)$ or bilaterally $(n=49,60 \%)$ on dedicated temporal bone CT scans. The median age for these 82 patients was 54 years, ranging from 1 to 91 years of age, and $57 \%(n=47)$ were men and $43 \%(n=35)$ women. Indications provided for CT scans noted hearing impairment in $52 \%$ of cases. Review of each patient's electronic medical record revealed that $88 \%$ of the study population had a history of hearing impairment at the time the CT was performed. The discrepancy between the two is attributable to the common scenario of incomplete medical histories provided in the indications for radiologic studies or provided indications that are associated with hearing loss without explicitly stating it.

Forty-one of the $82(50 \%)$ also had MR imaging of the diverticula available at the time of review. Of those patients, 22 (54\%) had corresponding fluid signal within the presumed IAC diverticulum and $19(46 \%)$ did not. Notably, 15/18 (83\%) patients showed fluid signal when the slice thickness of the MR imaging was $\leq 1 \mathrm{~mm}$, but only $7 / 23$ (30\%) showed fluid signal when the slice thickness was $3-5 \mathrm{~mm}$.

Twenty-eight (34\%) of the 82 patients with either unilateral or bilateral IAC diverticula also had otosclerosis. Bilateral IAC diverticula were observed in 20/28 (71\%) with otosclerosis in contrast to $29 / 54$ (54\%) patients without otosclerosis. The median size of the IAC diverticulum in patients without otosclerosis was $1.1 \mathrm{~mm}$ (IQR, $0.9-1.3 \mathrm{~mm}$ ) compared with ears with otosclerosis having a median size of $1.4 \mathrm{~mm}$ (IQR, $1.2-1.9 \mathrm{~mm})(P<.001)$; the maximum size from the right and left sides was used for patients with bilateral IAC diverticula. There was no correlation found between patient age and the size of the diverticula on either side (rightsided IAC diverticula: Spearman rank correlation coefficient, $0.12 ; P=.35$; and left-sided IAC diverticula: Spearman rank correlation coefficient, $-0.05 ; P=.70$ ).

Nine patients without audiometric data were excluded from subsequent analysis, leaving 73 patients with complete radiographic and audiometric data for both ears. Bilateral IAC diverticula were present in 42 (58\%) of these remaining patients, and unilateral IAC diverticula were present in 31 patients (42\%). For further subanalysis from these 31 patients, we excluded 8 with coexisting otosclerosis by radiologic assessment and 1 with otosclerosis by clinical assessment to avoid potential confounders that might increase the incidence of hearing loss and the possibility of underlying cavitary otosclerosis producing the focal lucency, leaving 22 patients. The mean age for this subset of 22 patients was $48.2 \pm 20.1$ years and included $14(64 \%)$ men and 8 (36\%) women. There were 13 (59\%) with right-sided diverticula and $9(41 \%)$ with left-sided diverticula, with a mean size of $1.0 \pm$ $0.2 \mathrm{~mm}$ (range, 0.6-1.4 $\mathrm{mm}$ ).

\begin{tabular}{|c|c|c|c|c|}
\hline \multirow[b]{2}{*}{ IAC Diverticulum } & \multicolumn{4}{|c|}{$\begin{array}{c}\text { Control Side } \\
\text { (No IAC Diverticulum) }\end{array}$} \\
\hline & Normal & $\mathrm{CHL}$ & MHL & SNHL \\
\hline Normal & 6 & 0 & 0 & 1 \\
\hline $\mathrm{CHL}$ & 1 & 1 & 1 & 0 \\
\hline MHL & 1 & 0 & 0 & 4 \\
\hline SNHL & 2 & 0 & 2 & 3 \\
\hline
\end{tabular}

This final group of 22 patients with isolated unilateral IAC diverticula and complete audiometric data for both ears provided 22 IAC diverticula and 22 nondiverticula internal controls. A comparison of hearing in the affected and unaffected ears is shown in Table 1. Within this subset, 6 patients had normal hearing in both ears and 11 patients had abnormal hearing (defined as CHL, MHL, or SNHL) in both ears. Four (18\%) patients had abnormal hearing in the ear with the IAC diverticulum, but normal hearing in the unaffected ear; in contrast, 1 (5\%) patient had normal hearing in the ear with the IAC diverticulum, but abnormal hearing in the unaffected ear $(P=.18$; McNemar test). The $P$ value for the comparison of the prevalence of normal/CHL versus MHL/SNHL between affected and unaffected ears was 65 .

Audiometric data comparing the ear with an IAC diverticulum with the contralateral side without an IAC diverticulum are shown in Table 2. The median air conduction pure tone average in the presence of an IAC diverticulum was $31 \mathrm{~dB}$ hearing level (HL) (IQR, 20-75 dB HL) compared with $20 \mathrm{~dB}$ HL (IQR, 9-41 dB $\mathrm{HL}$ ) in the control ear. The median difference between the two measurements, calculated as IAC minus non-IAC, was $4 \mathrm{~dB} \mathrm{HL}$ (IQR, 1-29 dB HL; $P=.056$ ). Median word recognition scores were 100 in both ears, resulting in a median difference of $0(P=$ $.55)$.

\section{DISCUSSION}

Previously published histopathologic studies of IAC diverticula, alternatively known as IAC cupping, demonstrated that these are well-marginated concavities along the anteroinferior wall of the IAC fundus with normal bone around the periphery of the concavity. ${ }^{1,2}$ The cases of isolated IAC diverticula in this study correlate well with the published histology, being characterized on CT by intact cortical, trabecular, and labyrinthine bone adjacent to the concavity and, in most cases with volumetric 3D T2-weighted imaging, by normal CSF extending into the diverticulum (Fig 1). Historically, IAC diverticula have been referred to as normal variants, with a warning not to confuse them with pathology. ${ }^{1,2}$ Despite the evidence in the literature characterizing the benign nature of these diverticula, a recent publication has found an association between these lesions and SNHL. ${ }^{4}$ In the current study, we did not identify a statistically significant association with SNHL or word recognition score loss. These findings corroborate our anecdotal clinical observation and prior pathology-based studies that showed isolated IAC diverticula (ie, those not associated with cavitary otosclerosis) should be considered benign normal variants.

The etiology of IAC diverticula or cupping remains uncertain. Possible explanations include developmental variations in the in- 
Table 2: Comparison of audiometric data in ears for patients with unilateral IAC diverticula in the absence of otosclerosis ( $N=22)^{a}$

\begin{tabular}{|c|c|c|c|c|c|}
\hline & No. ${ }^{b}$ & IAC Diverticulum & No IAC Diverticulum & Difference $^{c}$ & $P$ Value $^{\mathrm{d}}$ \\
\hline Bone conduction pure tone average & 22 & $22.5(13-41)$ & $17(8-35)$ & $1(-4-17)$ & .31 \\
\hline $\mathrm{BC} 250 \mathrm{~Hz}(\mathrm{~dB})$ & 20 & $17.5(7.5-25)$ & $12.5(7.5-20)$ & $2.5(-5-15)$ & .19 \\
\hline $\mathrm{BC} 500 \mathrm{~Hz}(\mathrm{~dB})$ & 21 & $20(10-40)$ & $15(10-25)$ & $5(0-20)$ & .14 \\
\hline $\mathrm{BC} 1000 \mathrm{~Hz}(\mathrm{~dB})$ & 20 & $22.5(10-40)$ & $15(5-27.5)$ & $0(-2.5-25)$ & .17 \\
\hline $\mathrm{BC} 2000 \mathrm{~Hz}(\mathrm{~dB})$ & 19 & $30(10-50)$ & $20(10-45)$ & $0(-10-15)$ & .50 \\
\hline $\mathrm{BC} 3000 \mathrm{~Hz}(\mathrm{~dB})$ & 18 & $25(7.5-47.5)$ & $20(10-55)$ & $0(-10-15)$ & .63 \\
\hline $\mathrm{BC} 4000 \mathrm{~Hz}(\mathrm{~dB})$ & 21 & $25(10-50)$ & $35(15-60)$ & $0(-5-5)$ & .97 \\
\hline Air conduction pure tone average & 22 & $31(20-75)$ & $20(9-41)$ & $4(1-29)$ & .06 \\
\hline $\mathrm{AC} 250 \mathrm{~Hz}(\mathrm{~dB})$ & 22 & $22.5(15-60)$ & $17.5(10-35)$ & $2.5(-5-20)$ & .19 \\
\hline $\mathrm{AC} 500 \mathrm{~Hz}(\mathrm{~dB})$ & 22 & $25(15-75)$ & $22.5(10-40)$ & $2.5(-5-20)$ & .30 \\
\hline $\mathrm{AC} 1000 \mathrm{~Hz}(\mathrm{~dB})$ & 22 & $27.5(15-75)$ & $20(5-40)$ & $5(0-30)$ & .06 \\
\hline AC $2000 \mathrm{~Hz}(\mathrm{~dB})$ & 22 & $32.5(15-70)$ & $20(5-50)$ & $10(0-30)$ & .10 \\
\hline AC $3000 \mathrm{~Hz}(\mathrm{~dB})$ & 20 & $37.5(15-60)$ & $20(12.5-62.5)$ & $5(-5-25)$ & .34 \\
\hline AC $4000 \mathrm{~Hz}(\mathrm{~dB})$ & 22 & $40(20-85)$ & $37.5(15-70)$ & $5(-5-40)$ & .22 \\
\hline Air-bone gap & 22 & $5(0-12)$ & $0(0-5)$ & $2(0-9)$ & .37 \\
\hline Word recognition (\%) & 17 & $100(85-100)$ & $100(95-100)$ & $0(-15-0)$ & .55 \\
\hline
\end{tabular}

Note:-AC indicates air conduction; BC, bone conduction.

a Data are median and IQR.

${ }^{\mathrm{b}}$ Summarized for a subset of patients with data for both ears.

C Calculated as IAC minus non-IAC.

${ }^{\mathrm{d}}$ Determined using Wilcoxon signed rank tests.

terface between the 14 periotic enchondral growth plates and the adjacent sites of secondary bone formation of the surrounding skull base. Considering that the IAC extends across the transition zone between primary enchondral bone and secondary bone formation, variations in this transition may explain the consistent location of the diverticulum in the anteroinferior margin of the IAC fundus. Bast and Anson, ${ }^{5}$ in their seminal temporal bone textbook, The Temporal Bone and the Ear, claimed that residual cartilaginous tissue, such as that seen in fissula ante fenestram, can be found elsewhere within the otic capsule and may explain the distribution of otosclerosis involving the labyrinthine bone. If the anteroinferior margin of the IAC fundus represents one of these residual cartilaginous rests, then its resorption may result in the concavity observed in this and other studies. Persistence of the cartilaginous rest might also serve as a focus of pericochlear otosclerosis, contributing to the appearance of what has been reported in the literature as cavitary otosclerosis involving this segment of the IAC fundus. ${ }^{6-8}$ Otospongiotic bone superimposed on a pre-existing congenital diverticulum might also explain the difference in diverticular size between the otospongiotic and nonotospongiotic groups.

The observed association between IAC diverticula and SNHL reported by Pippin et $\mathrm{al}^{4}$ could have arisen due to the potentially confounding effects of the inherent selection bias present when retrospectively examining a population of temporal bone CTs because these examinations have a much higher probability of being performed in patients with hearing loss. This inherent selection bias is exemplified by $88 \%$ of our patients with unilateral or bilateral IAC diverticula having either clinical concerns for or a reported history of hearing impairment in 1 or both ears at the time of their temporal bone CT scans. Our study design attempts to address the inherent selection bias issue by examining the subset of patients with unilateral IAC diverticula and without otosclerosis and by using the contralateral ear as an internal control. Because cochlear otosclerosis is defined as an SNHL due to involvement of the cochlear capsule, eliminating cases with cochlear otosclerosis should eliminate this as a potential confounder that could otherwise artificially increase the prevalence of SNHL in the study population. ${ }^{8}$ Using these criteria, we found that there is no statistically significant correlation of IAC diverticula with SNHL or decline in word recognition scores. Diverticula were shown to be present more often and were larger in patients with concurrent otosclerosis, but in patients without otosclerosis, though the IAC diverticula varied in size, there did not appear to be a pattern of progression with age. This observation, in combination with the benign-appearing osseous margins, as demonstrated on temporal bone histology (Fig 2), further supports the notion that IAC diverticula are congenital rather than acquired and do not progress with time.

This normal variant can usually be distinguished from cavitary otosclerosis, which classically involves the anteroinferior wall of the IAC fundus and occurs in conjunction with pericochlear and fenestral otosclerosis (Fig 3). ${ }^{6-8}$ An anterior wall IAC concavity with density greater than that in the adjacent CSF and with illdefined bony margins should raise concern for cavitary otosclerosis. Patients with cavitary otosclerosis will almost always have radiographic evidence of fenestral and/or retrofenestral otosclerosis in addition to audiometric evidence of a conductive or mixed hearing loss. Thus, finding a focal lucency in the anterior wall of the IAC should raise suspicion for cavitary otosclerosis if these findings are also present.

IAC diverticula are relatively common (approximately 5\% prevalence), and cavitary otosclerosis involving the anteroinferior wall of the IAC fundus is comparatively quite rare. ${ }^{4,6,8}$ Discerning the difference between the two is clearly of clinical importance. In profound SNHL secondary to pericochlear otosclerosis, the presence of cavitary otosclerosis may predispose to electrode array extrusion during cochlear implant insertion. ${ }^{7}$ The absence of concurrent otosclerosis, as was seen in most $(54 / 82,66 \%)$ of our patients, should be reassuring that such a finding does not represent the sequelae of disease. Furthermore, the presence of a wellmarginated lucency in the anteroinferior wall of the IAC fundus in the absence of surrounding otospongiotic bone should heavily favor an isolated IAC diverticulum over cavitary otosclerosis. 


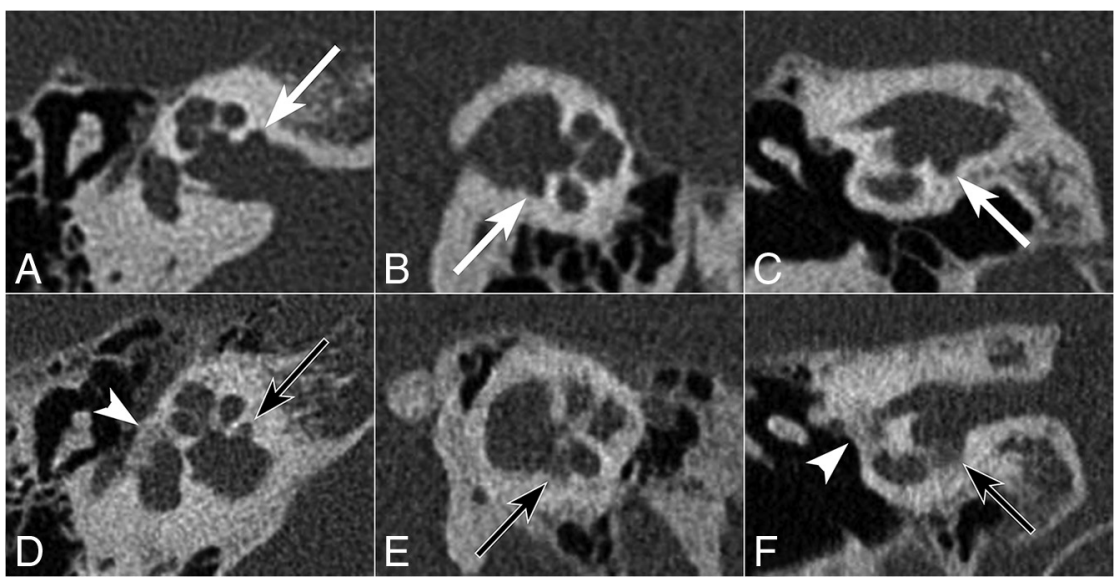

FIG 3. Right temporal bone axial ( $A$ and $D$ ), Pöschl ( $B$ and $E$ ), and coronal ( $C$ and $F$ ) views of an IAC diverticulum (upper row) versus cavitary otosclerosis (lower row). Note the well-demarcated margins of a lucent IAC diverticulum (white arrow) compared with the ill-defined spongiotic bone composing the otosclerotic focus along the anterior wall of the IAC (black arrow), with indistinct bony margins between this lesion and the basal turn of the cochlea ( $E$ and $F$ ). Also note the presence of fenestral otosclerosis (white arrowhead in $D$ and $F$ ).

Correlation with clinical presentation, surgical history, and audiometric findings also remains important in assessing the potential importance of this finding. In the absence of other temporal bone derangement or findings to support an alternative diagnosis, an isolated well-demarcated lucency in the anteroinferior wall of the IAC fundus likely represents a normal variant and is unlikely to be of clinical importance. Conversely, attributing SNHL to the presence of these diverticula could lead a clinician to overlook other treatable causes of SNHL, which could, in turn, lead to permanent hearing loss.

Our study is limited by its retrospective approach, the lack of a true healthy control group, minor variations due to the use of CT scanners with different slice intervals despite a common manufacturer, and the use of a single neuroradiologist for interpretation. The population size in relation to the power of the study and the observed effect sizes raises the question of whether we would have been able to observe the difference asserted by an even smaller previous study. ${ }^{4}$ However, this remains the largest study of this radiographic entity to date, and the findings are in agreement with historical observations. ${ }^{1,2}$ Furthermore, the observed differences in frequency-specific bone conduction thresholds (ie, SNHL) and word recognition between the involved ear and contralateral control ear were small (median difference ranged between 0 and $5 \mathrm{~dB}$ in bone conduction thresholds; median word recognition score difference, $0 \%$ ), suggesting that even if differences were found to be statistically significant in a study with much larger patient numbers, this small difference would still not be considered clinically relevant (Table 2). We have attempted to address some of the inherent weaknesses of a retrospective analysis and lack of true healthy controls with our case selection and use of internal controls. Based on our limited number of cases in which volumetric $\mathrm{CT}$ and MR imaging were available for review, further research comparing high-resolution MR imaging with audiometric data would be useful in corroborating our findings. Future studies might also attempt to objectively demonstrate which features best distinguish these IAC diverticula from cavitary otosclerosis in a population of patients with otosclerosis.

\section{CONCLUSIONS}

No statistically significant association was found between isolated IAC diverticula and SNHL or word recognition score loss. Our data support historical observations that these most likely represent a normal anatomic variant. ${ }^{1,2}$ In the absence of radiographic evidence of fenestral or pericochlear otosclerosis, the presence of IAC diverticula should bear no relationship to the clinical history of hearing loss.

\section{ACKNOWLEDGMENTS}

The authors acknowledge the assistance of Sonia Watson, $\mathrm{PhD}$, in editing the manuscript.

\section{REFERENCES}

1. Gulya AJ, Schuknecht HF. The inner ear. In: Gulya AJ, ed. Gulya and Schuknecht's Anatomy of the Temporal Bone with Surgical Implications. 3rd ed. New York: Informa Healthcare USA; 2007:168-69

2. Kollias SS. Imaging of the congenitally malformed temporal bone. In: Lemmerling M, Kollias SS, eds. Radiology of the Petrous Bone. Berlin: Springer-Verlag; 2004:121

3. Hoeberigs MC, Postma AA, Waterval JJ, et al. Prevalence of anterior internal auditory canal "diverticulum" on high resolution CT in patients with otosclerosis. In: Proceedings of the Radiological Society of North America Scientific Assembly and Annual Meeting, Chicago, Illinois; November 25-30, 2012

4. Pippin KJ, Muelleman TJ, Hill J, et al. Prevalence of internal auditory canal diverticulum and its association with hearing loss and otosclerosis. AJNR Am J Neuroradiol 2017;38:2167-71 CrossRef Medline

5. Bast TH, Anson BJ. The Temporal Bone and the Ear. Springfield: CC Thomas; 1949

6. Bou-Assaly W, Mukherji S, Srinivasan A. Bilateral cavitary otosclerosis: a rare presentation of otosclerosis and cause of hearing loss. Clin Imaging 2013;37:1116-18 CrossRef Medline

7. Makarem AO, Hoang TA, Lo WW, et al. Cavitating otosclerosis: clinical, radiologic, and histopathologic correlations. Otol Neurotol 2010; 31:381-84 CrossRef Medline

8. Makarem AO, Linthicum FH. Cavitating otosclerosis. Otol Neurotol 2008;29:730-31 CrossRef Medline 